\title{
Eficacia de la combinación de pamoato de oxantel y albendazol para el tratamiento de la trichiuriasis en niños
}

Efficay of a combination of oxantel pamoate and albendazole for the treatment of trichuriasis in childrem

Speich B y col. N Engl J Med 2014;370:610-20.

\section{Objetivo}

Comparar la eficacia y la seguridad de cuatro esquemas farmacológicos para el tratamiento de la infección por Trichuris Trichiura (resultado primario), Ancylostoma duodenale y Ascaris lumbricoides (resultado secundario).

\section{Diseño, lugar y pacientes}

Ensayo clínico aleatorizado doble ciego realizado en Pemba Tanzania durante 2012 en niños de 6 a 14 años de edad de dos escuelas. Fueron considerados elegibles, quienes arrojaron resultado positivo para $T$. trichiura $0 A$. duodenale en sus heces. Fueron criterios de exclusión la constatación de alguna enfermedad sistémica detectada a través de un interrogatorio y un examen clínico (p. ej. malaria clínica o esquistosomiasis hepatoesplénica).

\section{Intervención y medición de resultados principales}

Fueron asignados aleatoriamente a recibir uno de los siguientes cuatro tratamientos: 1) Pamoato de oxantel (PO) a una dosis de $20 \mathrm{mg} / \mathrm{kg}$ de peso corporal más $400 \mathrm{mg}$ de Albendazol (A); 2) $\mathrm{PO}$ en una dosis única de $20 \mathrm{mg} / \mathrm{kg}$ más placebo (PL); 3) A en una dosis única de 400 mg más PL; 4) M en una sola dosis de $500 \mathrm{mg}+\mathrm{PL}$. Cada niño recibió dos tabletas durante dos días consecutivos.

La eficacia se determinó a través de la proporción de niños sin huevos en las heces después del tratamiento.

\section{Resultados principales}

De los 900 niños pesquisados 480 fueron elegibles y aleatorizados. 472 fueron positivos para $\mathrm{T}$. trichiura, 456 para A duodenale y 309 para A lumbricoides. En 316 se documentó infección triple.

El tratamiento con PO fue un 31,2\% (IC95\% 22,5 a 40) más eficaz que los otros tres en los niños con infección por T. trichiura. Una única dosis de PO resultó significativamente más eficaz que una de $M(26,3 \%$ vs. $11,8 \%, p=0,01)$ y la monoterapia con A fue menos eficaz que la de $M(2,6 \%$ vs. $11,8 \%, p=0,02)$.

La tasa de curación en niños con infección leve fue de 39,0\% con PO+A, de $39,3 \%$ con monoterapia de PO, de $21,1 \%$ en los asignados a $M$ y de $4,5 \%$ en los asignados a $A$.

$\mathrm{PO}+\mathrm{A}$ y $\mathrm{PO}$ en monoterapia se asociaron con altas tasas de reducción de huevos en los niños con infección por T. trichiura: $96,0 \%$ (IC95 93,5 a 97,6) para el primero y 93,2\% (90 a 95,7) para el segundo. En el $84,9 \%$ de los niños con infección moderada o grave por T. trichiura se documentó curación o infección leve luego del tratamiento con $\mathrm{PO}+\mathrm{A}$.

\section{Conclusiones}

PO o sin A tiene una mostró una mayor eficacia en el tratamiento de $\mathrm{T}$. trichiura en comparación con monoterapia de $\mathrm{A} \circ \mathrm{M}$, sin que se observara una mayor incidencia de efectos adversos a las $24 \mathrm{~h}$ horas luego de la administración de la última dosis.

Fuente de financiamiento: Fundación Medicor y la Fundación Nacional de Ciencias de Suiza.

\section{Comentario}

Las infecciones por helmintos transmitidas por el suelo (ascaridiasis, ancilostomiasis y trichuriasis) se han generalizado y a menudo se producen de forma concomitante 1 . El objetivo primario del presente estudio fue evaluar el perfil de eficacia y seguridad de una combinación de PO y A (Zentel NR, GlaxoSmithKline) en niños infectados con T. trichiura. Las monoterapias con PO, A y M (Vermox, Johnson \& Johnson) sirvieron como comparadores y los autores comunicaron que la industria farmacéutica no tuvo injerencia en el desarrollo del estudio ni en el análisis de los datos.

\section{Conclusiones de las comentadoras}

Si bien este estudio cumple con todas las normas técnicas para asegurar un reporte transparente de los resultados, antes de aplicarlos a la población de Argentina vale tener en cuenta las siguientes consideraciones: 1) al no ser muy alta la tasa de curación con el régimen propuesto, podría incrementarse el desarrollo de resistencia a los antibióticos; 2) estas drogas no han sido aprobadas para su uso en humanos por agencias regulatorias como la Administración de Drogas y Alimentos de EE.UU. (en inglés FDA) y sí para animales como perros y gatos (deben comercializarse con la leyenda "Sólo para uso animal, mantener lejos de los niños"); mientras que en la agencia española sólo ha sido aprobado en humanos el uso de Pamoato de Pirantel y no así el $\mathrm{PO}^{2}$; 3) no hay suficientes ensayos clínicos controlados en la población infantil ${ }^{3}$ y no se conocen sus resultados en el largo plazo; 4) en Argentina no se dispone de PO.

Lidia Caballero [ Hospital Dr. Pedro Baliña, Posadas, Misiones. Argentina, lidia.gladis@gmail.com]

Gloria Córdoba Currea [ General Practice Section, University of Copenhagen ]

Caballero L y Córdoba Currea G. Eficacia de la combinación de pamoato de oxantel y albendazol para el tratamiento de la trichiuriasis en niños. Evid Act Pract Ambul.2014 17(4). Oct-Dic 126. Comentado de: Speich B y col. Oxantel pamoate-albendazole for Trichuris trichiura infection. Engl J Med 2014;370:610-20. PMID 24521107.

\section{Referencias}

1. Albonico $\mathrm{M}$ y col. Control strategies for human intestinal nematode infections. Adv Parasitol 1999; 42:277-341.

2. http://www.aemps.gob.es/cima/pdfs/es/p/49756/P_49756.pdf

3. WHO Model List of Essential Medicines for Children (2nd list, March 2010 update). Geneva: World Health Organization 2010.

4. Keiser J y col. Efficacy of Current Drugs Against Soil-Transmitted Helminth InfectionsSystematic Review and Meta-analysis. JAMA. 2008 ; 299 (16):1937-1948. doi:10.1001/jama.299.16.1937.

‡Pemba es una isla que forma parte del archipiélago de Zanzíbar, localizada a $50 \mathrm{~km}$ de la costa oriental de África, en aguas del océano Índico donde las helmitiasis son endémicas en su población infantil. 